Military Technical College Kobry El-Kobbah, Cairo, Egypt

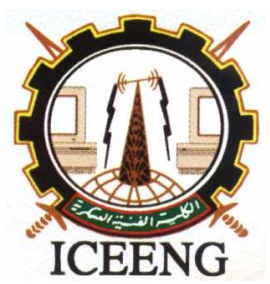

\author{
$6^{\text {th }}$ International Conference \\ on Electrical Engineering \\ ICEENG 2008
}

\title{
Realization of proposed optimum sonar detector based on digital signal processing techniques
}

$$
\text { By }
$$

\author{
K.H. Moustafa*
}

\section{Abstract:}

The proposed sonar detector will be illustrated in two cases: cross-correlator detector, and matched filter detector. In each case, we are interested to the output of the detector when the input signal is noisy free and due to signal contaminated by noise, which is assumed to be Gaussian noise. The complexity in the cross-correlator detector may be solved based on the matched filter structure, because of its less complexity and time calculation.

This paper also introduces a digital realization of a modified recursive tapped delay line integrator (transversal filter). Such a designed delay line based on analog techniques has a large size and suffers from instability. The digital implementation of this filter solves many of these problems. This paper also introduces a digital realization of a modified recursive SPMF (Single Pulse Matched Filter) and a digital realization of a modified recursive matched filter. So an introduced optimum sonar detector is implemented based on digital modified recursive matched filter.

\section{Keywords:}

Single Pulse Matched Filter, Modified recursive tapped delay line, and transversal filter. 


\section{Introduction:}

The radar or active sonar is a system that transmits a wave of known shape and receives the echoes returned by different obstacles or targets. The transmitted wave can be a pure frequency or its amplitude, phase, or frequency can be modulated. On reception, this wave must be amplified and analyzed in one way or another. This amplification has the effect of adding spurious noise to the received wave. This noise can generally be considered as white and Gaussian in the frequency band used. If the target is stationary, the received signal is therefore a noisy replica of the transmitted signal, with a delay corresponding to the target two-way path and modified by a complex coefficient (amplitude and phase) resulting from the attenuation due to propagation and reflection from the target. This complex coefficient is unknown, but its existence must obviously be taken into account by signal processing [1].

If the target has a constant range-rate motion, the Doppler effect generates further distortion of the transmitted signal by a frequency shift proportional to the radial velocity of the target and to the transmitted frequency. In general, the received signal in active radar or sonar can be modeled, to a first approximation, by a replica of the transmitted signal, which is distorted in a known manner when the set of parameters is known and multiplied by an unknown complex coefficient and to which Gaussian noise has been added [2]. The improvement of sonar system involves attempting to optimize these factors: [5]

- The transmitted signals (waveform, carrier frequency, transducer positioning, or, in passive systems, frequency bands used.

- The structure of the receiver in order to maximize the accuracy of target parameter estimation or the detection probability, given the characteristics of the noise and spurious signals.

The effectiveness of the present-day submarine depends upon its ability to remain undetected for long periods of time while it searches, tracks, or attacks from beneath the sea surface. Before a submarine can be attacked, it must be detected. In this paper, the detection performance is improved using the following processors:

- The digital SPMF which is introduced to improve the signal-to-noise ratio of a single pulse S-time compared to that achieved by analog filter, where $\mathrm{S}$ is the number of samples within the pulse.

- The digital transversal filter which is proposed to improve the signal-to-noise ratio of a train of $\mathrm{N}$-pulses by $\mathrm{N}$-time compared to that achieved by analog filter, where $\mathrm{N}$ is the number of pulses within the pulse repition period. 
- The digital matched filter, which is proposed to improve the signal-to-noise ratio of a train of N-pulses by SN times.

- The proposed modified detector for sonar based on digital modified recursive matched filter.

All of these processors are realized recursively and non-recursively.

\section{Improvement of Sonar Performance based on Digital Filters:}

Consider $s(t)$ is the signal transmitted by the transducer of the radar or the sonar. This signal is generally narrowband and can therefore be written in the form:

$$
s(t)=u(t) e^{j w 0 t}
$$

Where $u(t)$ is the complex envelope of the transmitted signal; ${ }^{w} 0$ is the angular frequency of the carrier [5]. Then, the signal received from a target located at range ${ }^{R_{0}}$, whose radial velocity is $\mathrm{v}$, is:

$$
A u\left(t-2 R_{0} / c\right) e^{-j \varphi} e^{j \omega_{0}(1+2 v / c) t}
$$

If $v / c<<1 \rightarrow B T<<d 2 v$, where $B$ is the signal bandwidth, $T$ is the signal duration, and $A$ is the received signal amplitude. This signal can also be written as:

$$
\begin{aligned}
& \alpha u\left(t-2 R_{0} / c\right) e^{j \omega_{0} 2 v t / c} e^{-j \varphi} e^{j \omega_{0} t} \\
& \alpha a(t, \theta) e^{-j \varphi} e^{j \omega_{0} t} \text { or again }
\end{aligned}
$$

Where $\theta$ is the vector of the parameters to be measured, equal to:

$$
\begin{aligned}
& \left(\begin{array}{c}
2 R_{0} / c \\
2 \omega_{0} v / c
\end{array}\right) \\
& a(t, \theta)=u\left(t-2 R_{0} / c\right) e^{j \omega_{0} 2 v t / c} \text { and }
\end{aligned}
$$

It is clear that the basic problem of sonar is to measure the time of arrival and the frequency of a signal. The two-way path of the transmitted wave generally has the following effects on the signal received by the radar or sonar [5].

- A delay $2 \mathrm{R}_{0} / \mathrm{C}$ ( due to the out-and- return propagation);

- A phase shift ( also to this two-way propagation); 
- A shift in the frequency received (Doppler Effect $f_{\mathrm{d}}=2 \mathrm{~V} / \lambda$, due to target closing).

Generally the impulse response of the filter which is matched to a certain received signal is the replica of its wave form. The intermediate frequency (IF) amplifier [6] is an approximation of a matched filter with the received reflected pulse from target. If the reflected echo from the target is a train of N-pulses, substantial improvement in target detectability can be achieved by integrating these pulses. Integration may be in the IF section of the receiver (predetection integration) or in the video section (post detection integration). Integration may also be used to eliminate the synchronous interference. In this case, the input signal is quantized, i.e. it takes one of two levels "0", or "1" depending on the comparison of the received signal amplitude with a threshold level. If the threshold level is low, the probability of false alarm increases while the probability of target detection decreases. The opposite situation occurs if this level is high. Usually, the threshold level is selected according to Neyman-Pearson optimization criterion [6] which increases a constant false alarm rate. The output of the integrator is compared with a second threshold level. This level corresponds to the output if M-pulses are received $[M \leq N]$ such that the decision that the target is true is issued if the integrator outputs exceeds this level, while if it is small, the received pulses are assumed to be noise or asynchronous jamming. This is known as M-out-of $\mathrm{N}$ criteria [4].

The impulse invariance method is a classical technique for designing digital filters [4]. Given a frequency characteristic, $H(f)$ an appropriate analog method is used to get the analog filter transfer function, $H(s)$ the best approximates $H(f)$. The digital filter transfer function $H(z)$ is obtained according to the following procedure in [9]. The digital filters are described in time and frequency domains.

The effect of the filter on the signal-to-noise ratio $[\mathrm{S} / \mathrm{N}]$ is described by the term" the improvement factor". It is defined as [8]:

$$
I=\frac{(S / N)_{\text {output }}}{(S / N)_{\text {input }}}
$$

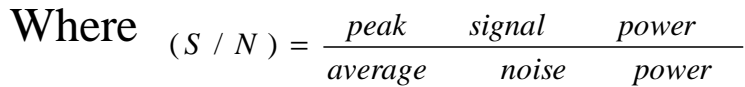

Assuming the input signal is rectangular pulses of amplitude $\mathrm{A}$ and input noise has a Gaussian distribution of standard deviation $\sigma$

- Single pulse matched filter (SPMF)

The matched filter impulse response is defined as [9]:

$$
h(t)=X^{*} \cdot\left(t_{o p}-t\right)
$$


Where $t_{o p}$ is an optimum delay that makes the filter causal, and $(*)$ denotes the complex conjugate. For the post detection processing $X(t)$ is real and $h(t)$ is simply the image of the input signal. For $X(t)$ being the rectangular pulse defined as:

$$
X(t)=\left\{\begin{array}{cc}
1 & 0 \leq t \leq t_{P} \\
0 & O . W
\end{array}\right.
$$

The single pulse matched filter (SPMF) is found to have the following impulse response:

$$
h(t)=\left\{\begin{array}{cc}
1 & 0 \leq t \leq t_{P} \\
0 & O . W
\end{array}\right.
$$

The analog implementation of equation (11) is shown in Figure (1).

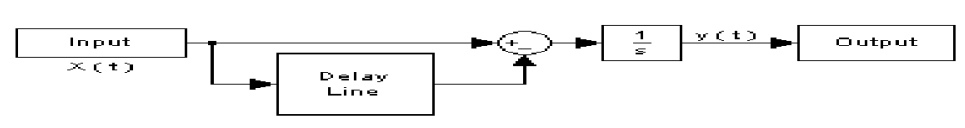

Figure (1): Analog realization of SPMF

The output of the filter $y(t)$ is the autocorrelation function, which in our case become [4]:

$$
y(t)=\left\{\begin{array}{cc}
t & 0 \leq t \leq t_{p} \\
2 t_{p}-t t_{p} & \leq t \leq 2 t_{p} \\
0 & O . W
\end{array}\right.
$$

The impulse response of a SPMF is shown in Figure (2)



Figure(2): Impulse response of a SPMF

For the digital realization of this filter, if $f_{S}$ is the sampling frequency, then there are $\mathrm{S}$ samples during the pulse duration $t_{P}$, where $\mathrm{S}$ is given by:

$$
S=t_{P} \cdot f_{S}
$$

The corresponding impulse response is given by:

$$
h\left(n T_{S}\right)=\left\{\begin{array}{cc}
1 & n=0,1, \ldots, s-1 \\
0 & \text { o.w. }
\end{array}\right.
$$

The corresponding $H(Z)$ is given by [4]:

$$
H(Z)=1+Z^{-1}+Z^{-2}+\ldots .+Z^{-(s-1)}
$$

The frequency response of the non-recursive SPMF is given by [4]:

$$
H\left(e^{-j W}\right)=1+e^{-j W T_{S}}+e^{-2 j W T_{S}}+\ldots .+e^{-j(S-1) W T_{S}}
$$


The time domain response of this filter is also given by:

$$
y\left(n T_{S}\right)=X\left(n T_{S}\right)+X\left((n-1) T_{S}\right)+X\left((n-2) T_{S}\right)+\ldots+X\left((n-(s-1)) T_{S}\right)
$$

Non-recursive realization of digital SPMF with $S=10$ based on SIMULINK subroutine at MATLAB is shown in Figure (3)

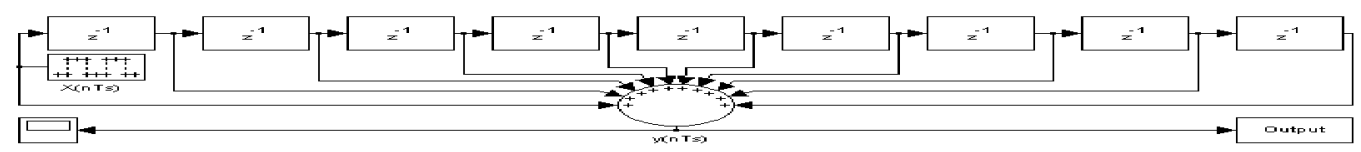

Figure (3): Realization of non-recursive digital SPMF for $S=10$.

The frequency response and time domain response of non-recursive SPMF for $S=10$ and 20 are shown in Figure (4) and Figure (5) respectively.

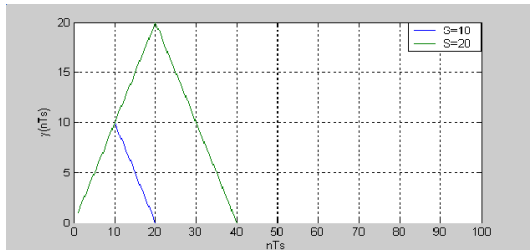

Figure(5): Time domain response of nonrecursive digital $S P M F$ for $S=10, S=20$

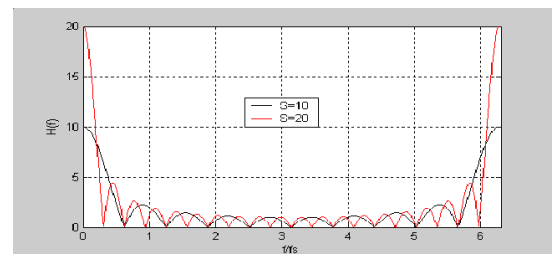

Figure(4): Frequency response of nonrecursive digital $S P M F$ for $S=10, S=20$

It is clear that as $\mathrm{S}$ increases, the peak of the autocorrelation function increases. The non-recursive digital SPMF is always stable. Meanwhile, it suffers from the low speed of processing and the complexity of realization. The digital SPMF is used to improve the receiver performance with respect to a single received pulse. The improvement depends on the number of samples within the pulses. This number depends on both the pulse duration $\delta$, and sampling period $\mathrm{T}_{\mathrm{s}}$. In case of $\left[\delta=100 \mathrm{~ms}, T_{s}=10 \mathrm{~ms}\right.$ ], the number of samples within one pulse is ten. The transfer function of recursive digital SPMF is expressed as [9]:

$$
H(Z)=\left(1-Z^{-S}\right) /\left(1-Z^{-1}\right)
$$

The corresponding realization is shown in Figure (6)

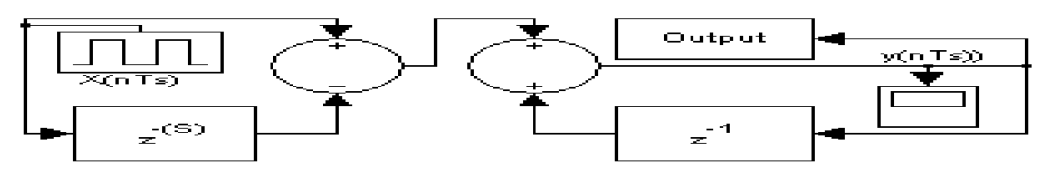

Figure(6): Recursive realization of digital SPMF

The disadvantage of recursive realization of digital SPMF is that it is marginally stable. The stabilization of such a filter is achieved by multiplying the feed back recursive branch by a stabilization coefficient $(\mathrm{A}<1)$, then the stabilized function is given by:

$$
H(Z)=\left(1-Z^{-S}\right) / A \cdot\left(1-Z^{-1}\right)
$$


The realization of stabilized recursive digital SPMF is shown in Figure (7)

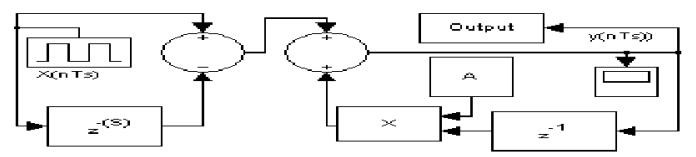

Figure(7): Stabilized recursive digital SPMF

The frequency response and time domain response of the stabilized recursive digital SPMF for different stabilization coefficients are calculated. Figure (8) and Figure (9) shows the frequency and time domain response of the stabilized recursive digital SPMF for $S=10$ and $A=1.0,0.9,0.8$ respectively.

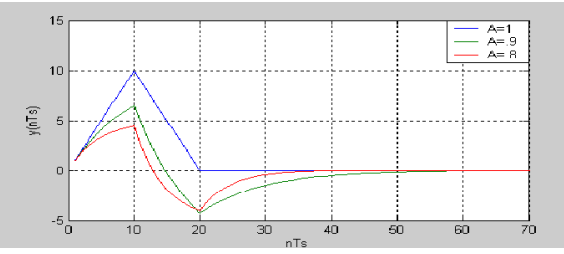

Figure (9): Time domain response of the stabilized recursive digital SPMF for $S=10$, and $A=1.0,0.9$, and 0.8

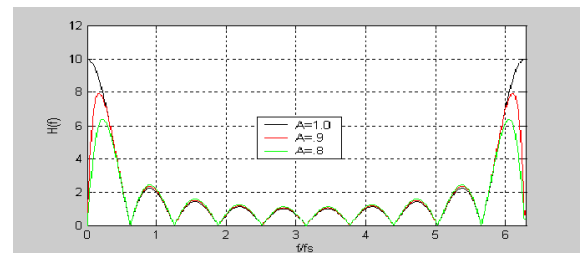

Figure (8): Frequency response of the stabilized recursive digital SPMF for $S=10$, and $A=1.0,0.9$, and 0.8

It is clear that, the multiplication by the stabilization coefficient $(A)$ slightly distorts the space of the output of the filter. This distortion increases as the value of the multiplication coefficient decreases. We can eliminate this distortion by introducing another multiplication factor. Then equation (18) tens to:

$$
H(Z)=\frac{1-\left(A Z^{-1}\right)^{S}}{1-A Z^{-1}}
$$

The factor $\left(1-A Z^{-1}\right)$ is one of the numerator factors and, so it can be calculated with the one of zeros of denominator. The realization of modified recursive digital SPMF is shown in Figure (10)

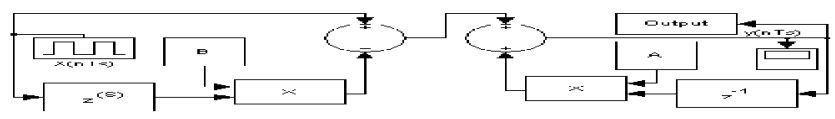

Figure(10): Modified recursive digital SPMF

For the case $S=10$, the frequency and time domain response of the modified recursive digital SPMF for different multiplication factors: $A=1.0,0.9,0.8$ are shown in Figure (11) and Figure (12) respectively.



Figure(12): Time domain response of the modified recursive $S P M F$ for $S=10$, and $A=1.0,0.9$, and 0.8

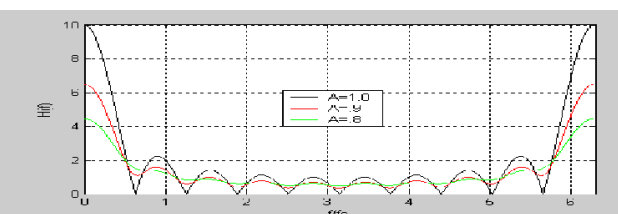

Figure(11): Frequency response of the modified recursive $S P M F$ for $S=10$, and $A=1.0,0.9$, and 0.8 
The $(\mathrm{S} / \mathrm{N})$ ratio of SPMF in which the number of samples within the pulse is $S$, is given by:

$$
(S / N)_{\text {input }}=\frac{A^{2}}{\sigma^{2}}
$$

The peak output of this filter is found to be:

$$
y(n)_{\text {pak }}=S A
$$

The average output noise power, $\overline{m_{0}^{2}}$ of the filter is given by:

$$
\overline{m_{0}^{2}}=S \sigma^{2}
$$

Finally, the output $(S / N)$ of the filter is given by:

$$
(S / N)_{\text {ouput }}=(A S) /\left(S \sigma^{2}\right)
$$

Then, the improvement factor of Digital SPMF is given by:

$$
I=S
$$

\section{- Digital Transversal filter}

The processing of a train of $\mathrm{N}$-video pulses implies the utilization of a transversal filter (tapped delay line). The analog realization of a transversal filter requires a tapped delay line with (N-1) sections; each has a delay time of $T_{r}=Z^{-1}$, (the pulse repetition period of a sonar system). The realization of non-recursive digital transversal filter is shown in Figure (13).



Figure(13): Non-recursive realization of the digital transversal filter for $N=10$

Figure (14) shows the frequency response of the non-recursive digital transversal filter for $L=2$, the pulse repition period, and $N=10$. Figure (15) shows the time domain response of the non-recursive digital transversal filter for $N=10, L=100$, and $S=10$. .

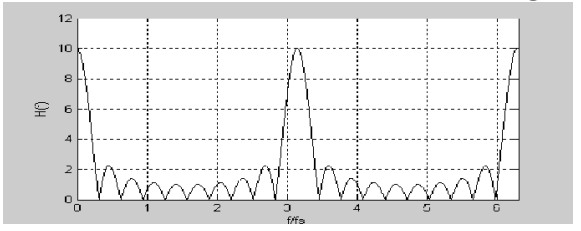

Figure(14): Frequency response of a non-recursive digital transversal filter for $N=10$, and $L=2$

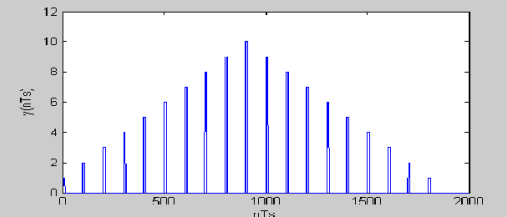

Figure(15): Time domain response of a non recursive digital transversal filter for $N=10, L=100$ and $S=10$

Similar to digital SPMF case, the modified transfer function of a digital recursive transversal filter is given by:

$$
H(Z)=\frac{1-\left(A Z^{-L}\right)^{N}}{1-A Z^{-L}}
$$

The realization of a modified recursive digital transversal filter is shown in Figure(16) 


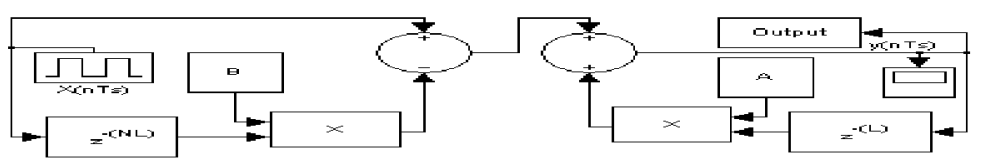

Figure(16): Realization of a modified recursive digital transversal filter

The frequency response of a modified recursive digital transversal filter for $L=2, N=10$, $A=0.98,0.90,0.80$ is shown in Figure (17). The time domain response of a modified recursive digital transversal filter for $L=90, N=10$, and $A=0.95$ is shown in Figure (18).

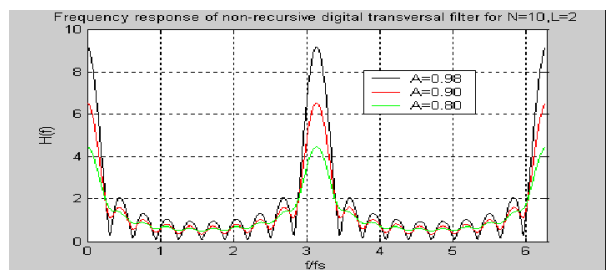

Figure (17): Frequency response of a modified recursive digital transversal filter for $L=2, N=10, A=0.98,0.90,0.80$

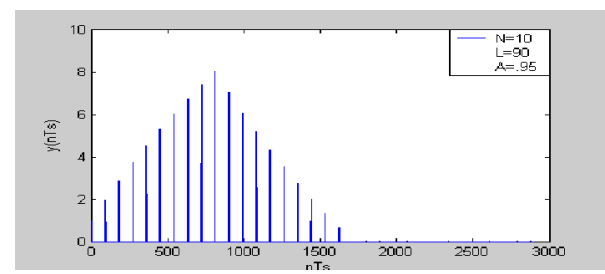

Figure (18): Time domain analysis of a modified recursive digital transversal filter for $N=10, L=100$ and $S=10$

The peak output of this filter is found to be:

$y(n)_{\text {peak }}=N A$

The average output noise power $\overline{m_{0}^{2}}$ of this filter is given by:

$$
\overline{m_{0}^{2}}=N \sigma^{2}
$$

The output $(S / N)$ of the filter is given by:

$$
(S / N)_{\text {output }}=\left(A N^{2}\right) /\left(N \sigma^{2}\right)
$$

The improvement factor of binary integration is given by:

$$
I=N
$$

\section{- Digital matched filter}

For the groups of N-video pulses, the matched filter consists of a transversal filter for the integration of N-pulses, cascade with a SPMF. The SPMF may b used before or after the transversal filter [4]. The non-recursive realization of a digital matched filter for $\mathrm{S}=10$ and $\mathrm{N}=10$ is shown in Figure (19).

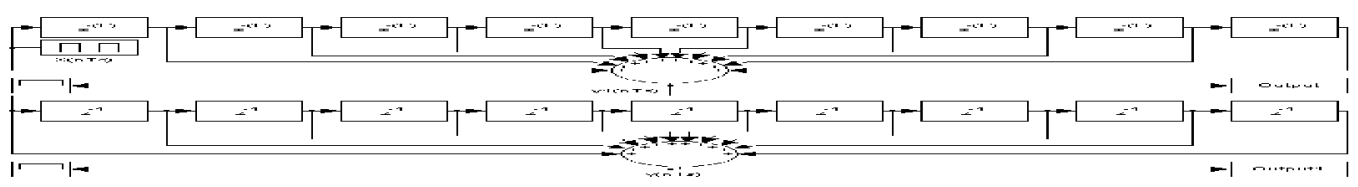

Figure (19): Non-recursive realization of a digital matched filter for $S=10$ and $N=10$

When the stabilization coefficient is considered, the frequency response and timedomain response of a modified recursive digital matched filter for $N=10, S=10$, and $L=40$ are shown in Figure (20) and Figure (21) respectively. 


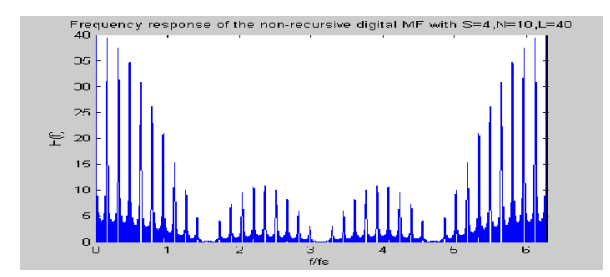

Figure (20): Frequency response of a modified recursive digital matched filter for $N=10, L=40$ and $S=10$

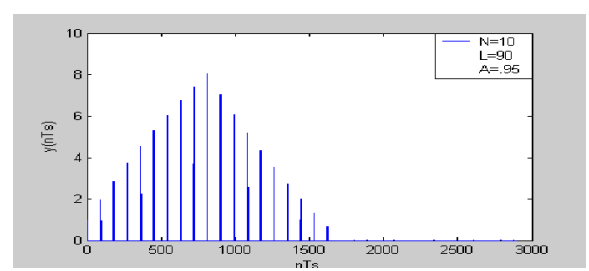

Figure(21): Time domain response of a modified recursive digital matched filter for $N=10, L=40$ and $S=10$

The peak output of the digital matched filter is found to be:

$y(n)_{\text {peak }}=(N S) \cdot A$

The average output noise power, $\overline{m_{0}^{2}}$ of this filter is given by:

$\overline{m_{0}^{2}}=N S \sigma^{2}$

The output $(S / N)$ of this filter is given by:

$$
(S / N)_{\text {ouput }}=(A N S)^{2} /\left(N S \sigma^{2}\right)
$$

Finally, the improvement factor of modified recursive digital matched filter is given by:

$$
I=N S
$$

\section{Realization of an optimum sonar detector:}

The problem of detecting a signal $s(t)$ in noise $n(t)$ consists of choosing between two hypotheses, depending on the received signal $r(t)$ :

$$
\begin{aligned}
& H_{1}: r(t)=s(t)+n(t) \\
& H_{0}: r(t)=n(t)
\end{aligned}
$$

Various criteria can be used; the most natural, corresponding to the approach actually used in practice, consists of considering two different types of error can be made:

- Deciding that there is a target $\left(H_{1}\right)$ when only noise is present $\left(H_{0}\right)$ : this is a false alarm, with probability $P_{f a}$ for a given decision rule;

- Deciding that there is no target $\left(H_{0}\right)$ when hypothesis $H_{1}$ is in fact true: this is a non detection of probability $P_{n d}$.

It is clear that in practice, the two errors are antithetical. Minimizing the probability of one of these errors will automatically increase the probability that the other will occur. The practical criterion used, that of Neyman-Pearson, consists of setting the false alarm probability $P_{f a}$ to a specified value $\alpha$ and minimizing the non detection probability [5]. The Neyman-Pearson criterion is used as an optimization criterion to maximize the detection probability at a fixed false alarm probability. Generally, the targets will be detected against a background of white Gaussian noise. 
For an efficient operation of sonar, the order of the probability of false alarm in sonar case must be varying from $10^{-3}$ to $10^{-6}$, and the probability of detection must be on the order of 0.5 to 0.9 at maximum range [2]. The detector based on this criterion is called optimum detector. Figure (22)(a) shows the structure of an optimum detector. This detector comprise consecutively a correlation with a replica of the expected signal (for each possible parameter $\theta$ ), quadrature detection, normalization with respect to the replica power, and comparison of these values with a threshold, defined according to the required false alarm probability [5].

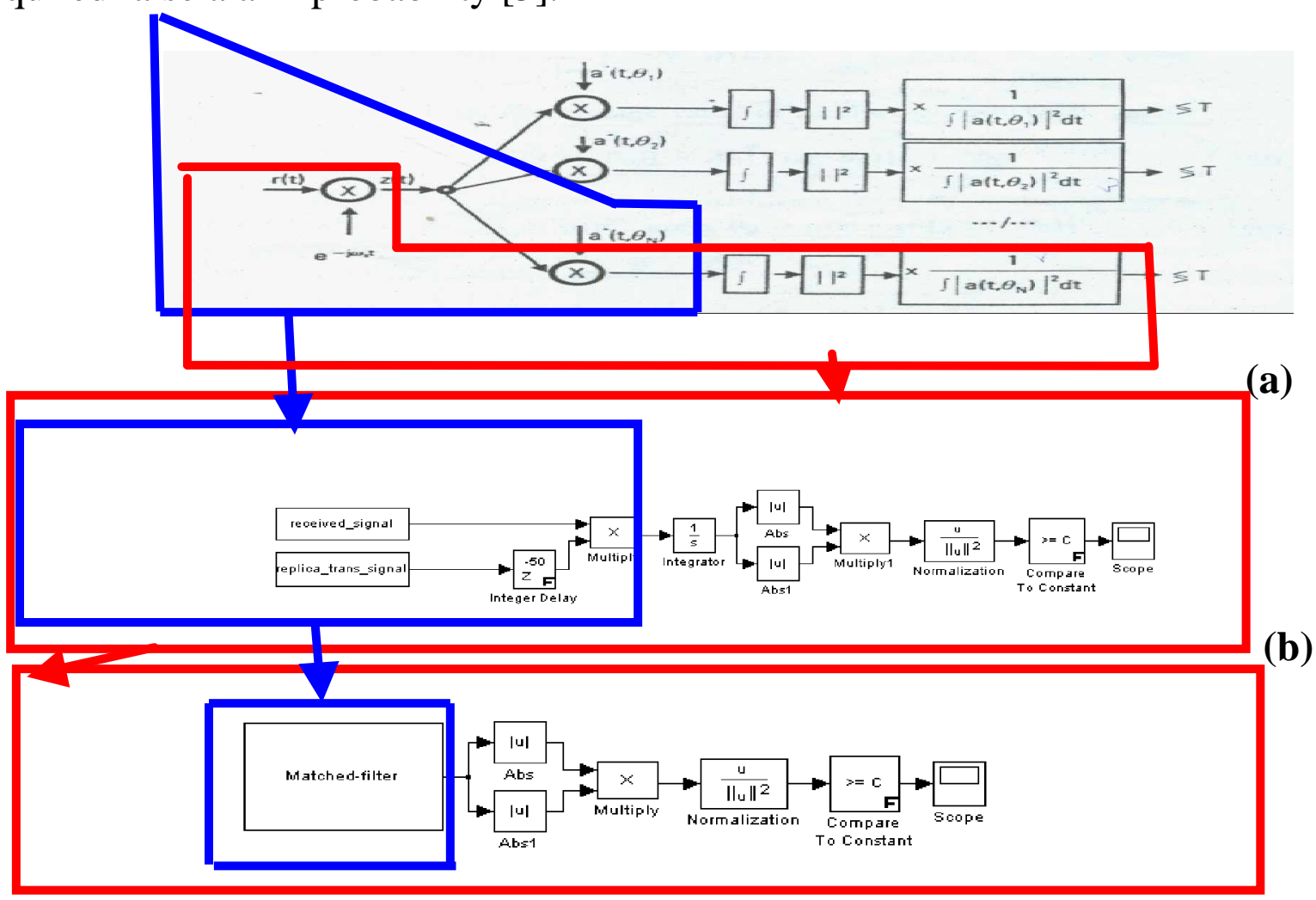

(c)

Figure (22): Structure of optimum receiver

This work introduces two models of sonar detector. The first model is the crosscorrelator detector shown in Figure (22) (b), and the second one is a modified recursive digital matched filter detector shown in Figure (22) (c). In each case, we are interested to the output of the detector when the input signal is noisy free and due to signal immersed in noise, which is assumed to be Gaussian noise. Models of the receiver structures are implemented using SIMULINK subroutine in MATLAB program. The input to the detector are excited as M-files using MATLAB program. The crosscorrelator detector needs a set of channels corresponding to time delays expected from the echo target. For each time delay there is an individual channel which leads to more complexity in implementation. In matched filter detector, instead of multiplying the input signal with a stored replica of the transmitted signal, the received signal is 
multiplied with itself delayed in time. The complexity in cross-correlator detector may be solved based on matched filter, because of its less complexity and time calculation. So our work dealing only with one channel of an optimum detector. This channel corresponds to the expected target echo delay which is chosen to be $50 \mathrm{~ms}$. This channel is surrounded by a dashed line shown in Figure (22) (a). For two sonar detector models, the input represents a practical sonar system [3] which is assumed to be a train of four rectangular pulses $(N=4)$, each with a $10 \mathrm{~ms}$ pulse width $S=10, L=100 \mathrm{~ms}$ pulse repetition period, and 10 samples within each pulse.

\section{Realization of Cross-correlation sonar detector:}

\section{a-Case of noise free signal}

The input and output of the cross-correlator detector is shown in Figure (23) and Figure (24) respectively

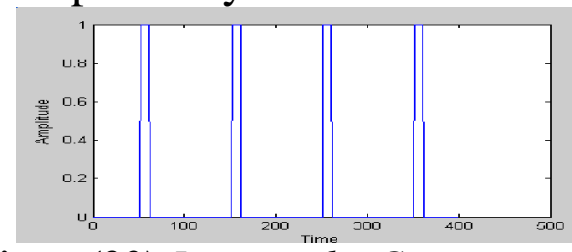

Figure(23): Input to the Cross-correlator with $\mathrm{N}=4, \mathrm{~S}=10 \mathrm{~ms}$, and $\mathrm{L}=100 \mathrm{~ms}$

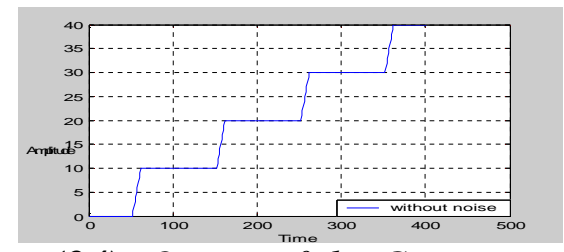

Figure(24): Output of the Cross-correlator for an input of $\mathrm{N}=4, \mathrm{~S}=10 \mathrm{~ms}$, and $L=100 \mathrm{~ms}$

\section{b-Case of signal contaminated by a Gaussian noise}

The cross-correlator detector is excited by a signal plus a Gaussian noise as shown in Figure (25), while the corresponding output is shown in Figure (26).

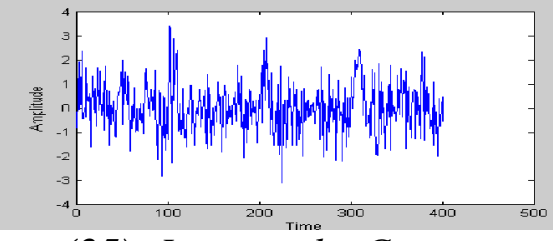

Figure(25): Input to the Cross-correlator with $\mathrm{N}=4, \mathrm{~S}=10 \mathrm{~ms}, \mathrm{~L}=100 \mathrm{~ms}$, and contaminated by Gaussian noise

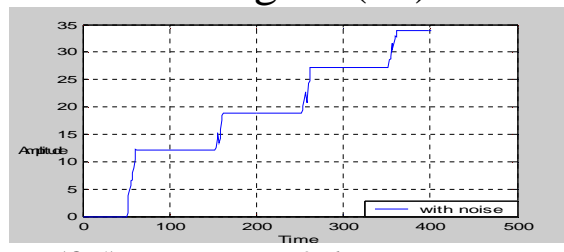

Figure(26): Output of the Cross-correlator for an input of $N=4, S=10 \mathrm{~ms}$, and $L=100 \mathrm{~ms}$ contaminated by Gaussian noise

To check the performance of proposed modified sonar cross-correlator detector under a Gaussian noise, a comparison between the performance of the sonar detector in the absence and the presence of a Gaussian noise is shown in Figure (27).

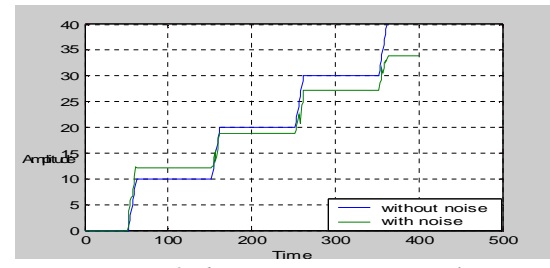

Figure (27): Comparison between outputs of the Cross-correlator for $\mathrm{N}=4, S=10 \mathrm{~ms}, L=100 \mathrm{~ms}$, in the presence and absence of a Gaussian noise 


\section{Realization of a modified recursive digital matched filter sonar detector:}

The matched filter performs an autocorrelation function between the received signal and a replica of the transmitted signal stored in its frequency response. But the cross correlation function is mathematically equivalent to the autocorrelation function and differs from each other in practical implementation way [7]. This means that the crosscorrelator detector described above may be replaced by a modified recursive digital matched filter. This digital filter is also excited by a train of four rectangular pulses $(\mathrm{N}=4)$, each with a $10 \mathrm{~ms}$ pulse width $(\mathrm{S}=10), \mathrm{L}=100 \mathrm{~ms}$ pulse repetition period, and 10 samples within each pulse.

\section{a-Case of noise free signal}

The input and output of the digital matched filter detector is shown in Fig.28 and Fig.29 respectively

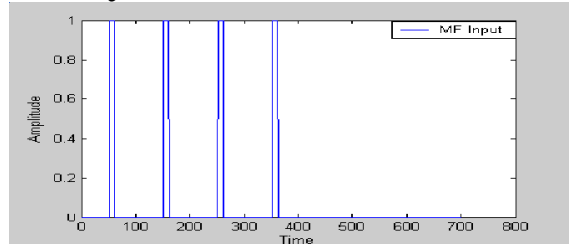

Figure (28): Input to the digital matched filter with $\mathrm{N}=4, S=10 \mathrm{~ms}$, and $L=100 \mathrm{~ms}$

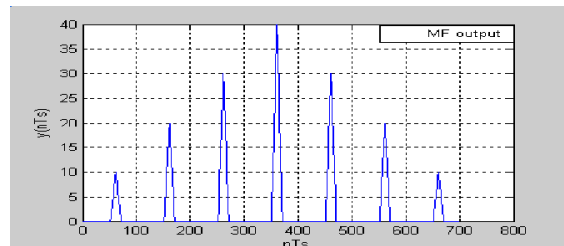

Figure (29): Output of the digital matched filter for an input of $\mathrm{N}=4, \mathrm{~S}=10 \mathrm{~ms}$, and $L=100 \mathrm{~ms}$

\section{b-Case of signal contaminated by a Gaussian noise}

The digital matched filter detector is also excited by a signal plus a Gaussian noise as shown in Fig.30, while the corresponding output is shown in Fig.31.

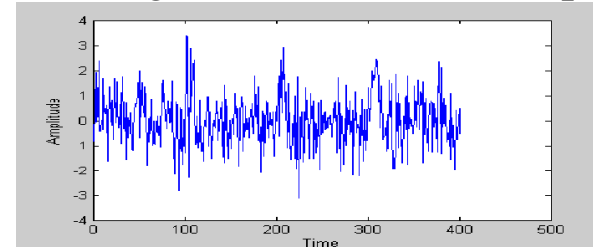

Figure (30): 30 Input to the digital matched filter with $N=4, S=10 \mathrm{~ms}, L=100$ ms, and contaminated by Gaussian noise

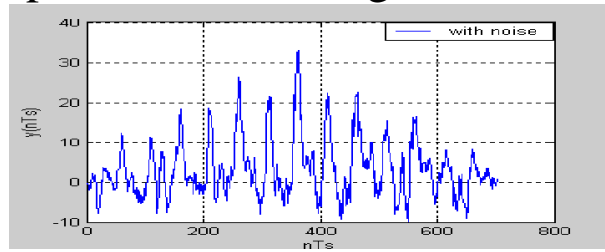

Figure (31): Output of the digital matched filter for an input of $N=4, S=10 \mathrm{~ms}$, and $L=100$ ms contaminated by Gaussian noise

To check the performance of proposed modified sonar matched filter detector under a Gaussian noise, a comparison between the performance of the sonar detector in the absence and the presence of a Gaussian noise is shown in Figure (32).

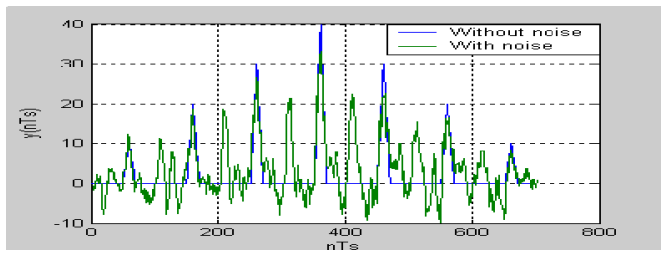

Figure(32): Comparison between outputs of a modified recursive digital matched filter for $N=4$, $S=10 \mathrm{~ms}, L=100 \mathrm{~ms}$, in the presence and absence of a Gaussian noise 
From results obtained above, when the signal is a noisy free, the cross-correlator detector performance is identical to that performance of the digital matched filter one. The cross-correlator needs a set of channels corresponding to the number of time delays expected from the echo target. For each time delay there is an individual channel which leads to more complexity in implementation. In matched filter, instead of multiplying the input signal with a stored replica of the transmitted signal, the received signal is multiplied with itself delayed in time. The complexity in the cross-correlator structure may be solved based on digital matched filter. Finally, it is clear that the modified recursive digital matched filter is a better choice to be proposed in sonar detector structure.

\section{Conclusion:}

One of the most common techniques used in the processes of detection of underwater targets with ultrasonic signals is the matched filter. The matched filter theory is based on knowledge of the exact shape of the pulse to be detected and the filter is designed so that it maximizes the SNR between the received signal and the delayed replica of the transmitted signal. Besides the nice property of increasing the SNR of its output when a signal is detected, the matched filter is also optimum in maximizing the probability of detection.

Improving performance of the underwater detection is achieved through improving sonar detectability by increasing the SNR either by using the digital single pulse matched filter or by using the digital delay line integrator (transversal filter) or by cascading both of them.

\section{References:}

[1] A. D. Waite, "Sonar for practicing engineering", Ashley Waite 2002

[2] Francois Le Chevalier, "Principles of radar and sonar signal processing", 2002.

[3] R.Frieden, "Principles of Naval Weapons systems", United States Naval Institute Press, 1985.

[4] B. A. Shenoi, "Introduction to DSP and filter design", 2006

[5] "Sonar Modeling Handbook, Volume 1. Modeling Procedures and Environments" .DRA/ Mar TM (USSF) 91172 (original version Jan. 1992, most recent update Dec. 1994), UK Defence Research Agency.

[6] Difranco, J.V., "Radar Detection", Artech House, 1980.

[7] [13] Skolnk, "Introduction to radar system." 2001.

[8] Paul A. Lynn, "Radar Systems", Macmillan education Ltd., 1987

[9] A. Antoniou. "Digital filters, Analysis and Design", 1979. 